\title{
Sperm Surface Antibodies: IUI vs. IVF Treatment
}

\author{
Afaf Felemban MD*, Seham M. Hassonah MD, Najla Felimban KD, Hadeel Alkhelb MD, Samar Hassan MD, \\ Fahad Alsalman MD \\ Department of Obstetrics and Gynecology, Riyadh, Saudi Arabia
}

*Corresponding Author: Dr. Afaf Felemban, Department of Obstetrics and Gynecology, King Saud bin AbdulAziz University for Health Sciences, Riyadh, Saudi Arabia, E-mail: fillimbana@ngha.med.sa

Received: 14 August 2018; Accepted: 20 August 2018; Published: 24 August 2018

Abstract

Objective: To compare the results of treating male immunological infertility using two different treatment options. Intrauterine Insemination (IUI) versus In-virto Fertilisation (IVF).

Design: Retrospective Study

Setting: IVF/Reproductive Endocrinology Unit, King AbdulAziz Medical City, Riyadh, Saudi Arabia.

Material and methods: Between year 1999 and 2005, 56 patients were recruited. Only patients with history of primary infertility and without any major cause of infertility such as tubal factor or anovulation were included. For all patients, the male partners had proven moderate to severe positive Mixed Antiglobin Reaction (MAR) test (IgG) (40-100\%). Samples were collected by masturbation and examined according to the World Health Organization (WHO) criteria except for the morphology the strict Tygerberg criteria was followed. Samples were washed with Gradients or Swim-up. Couples were counselled for IVF; however, if they opted for IUI their choice was respected. The two treatment options were: IUI for a maximum of three cycles (Group I n=37 patients, who went for 56 cycles) or IVF (Group II, n=19 patients who went for 23 cycles).

Primary Outcome: Pregnancy rate.

Results: Mean BMI $30.68 \pm 5.32$ in (Group1) and $30.96 \pm 5.65$ in (Group II). Group I (IUI) 6 patients became pregnant giving a pregnancy rate (PR)/cycle of $11 \%$ and a PR/patient of 16.2\%.Group II (IVF), 10 patients conceived giving a PR/cycle $43.5 \%$ and a PR/patient of 52.6\% (P-value < 0.05). 
Conclusion: Both the techniques of IUI and IVF revealed high pregnancy rates in the treatment of infertility due to sperm surface antibody. Ovarian hyperstimulation followed by IUI is much less invasive procedure for the patient than IVF. On the other hand, looking at the cost of HMG-IUI and IVF treatment cycle, we can support the super ovulation/IUI as the first option in the male immunological subfertility.

Keywords: Intrauterine Insemination; In-virto Fertilisation; Antisperm; Anti Sperm Antibodies

Abbreviations: ART-Assisted Reproductive Technology; ASA-Anti Sperm Antibodies; BMI-Body Mass Index; ICSI-IntraCytoplasmic Sperm Injection; IUI-Intrauterine Insemination; IVF-In-Virto Fertilisation; MAR-Mixed Antiglobin Reaction; OI-Ovulation Induction; PR-Pregnancy Rate; WHO-World Health Organization; ROSReactive Oxygen Species; SSA-Sperm Surface Antibody

\section{Introduction}

One in five reproductive age couples in the United States is infertile. Despite adequate medical evaluations, approximately $15 \%$ of them have unexplained infertility [1]. The mounting evidences support immunomodulation of fertility in many of those couples. One major aspect of this immunomodulation is the presence or absence of anti sperm antibodies (ASA) [2], but the clinical significance of ASA in male infertility remains unclear.

Most studies demonstrate a clear association between sperm surface antibodies and the fertility potential of the male, ASA found in semen are usually immunoglobulins of the IgG or IgA iso- type that are directed to various sites of the spermatozoa [3]. From a physiological point of view, immunological infertility due to sperm surface antibodies can result from the effect on sperm transport, the destruction of gametes, acrosome reaction abnormalities by inhibition of sperm-zona pellucida binding or by prevention of embryo cleavage and early development of the embryo [4]. Surprisingly spermatogenesis is apparently not affected by the presence of IgG or IgA ASA. However, sperm density and or morphology are not influenced by the presence of these antibodies [5]. A precise estimate of ASA prevalence is limited by the methods used for detection and their subsequent interpretation. Numerous methodologies are used to detect ASA. Each has distinct advantages and disadvantages [6]. Antibodies on the sperm surface can be detected by immunological assays using secondary, Ig class-directed antibodies that are coupled to beads. The percentage of sperm adhering to the beads reflects in a semi-quantitative manner the presence of antisperm antibodies.

We currently use Mar-test kits (FertiPro N.V. Industrie Park Noard 32, 8730 Beernem, Belgium) to detect antisperm IgG in semen. Positive samples are subsequently tested for antisperm IgA, which is more significant clinically, but rarely occurs without associated IgG. Therefore, the test anti-sperm IgG antibodies is sufficient for the first screening procedure. Concerning therapy several strategies are used in an effort to improve the potentially deleterious effects of ASA-mediated infertility. There are three basic strategies including: (1) methods to decrease ASA production, (2) methods to remove ASA already bound to sperm, and (3) Assisted Reproductive Technology 
(ART). Each of these strategies theoretically reduces gamete exposure to ASA, resulting in improved gamete function. Two methods used to reduce ASA production include condoms and systematic corticosteroids treatment [7].Clinical benefit after immuno suppression with steroids is only achieved after at least 6 months of therapy [8]. At the same time the use of antibiotics is only worthwhile if spermatozoa autoimmunity is caused by genitourinary infection.

Currently, the use of intrauterine insemination (IUI) after ovulation induction (OI) and assisted reproductive technology [In-vitro fertilization (IVF) with or without high insemination technique and intracytoplasmic sperm injection (ICSI)] are more popular and probably more effective. The reported average success for IUI in couples with male factor anti-sperm antibodies is $10-20 \%$. So our study is a retrospective study to compare success rates after two different treatment protocols for couples with male immunological infertility, namely IUI super ovulation versus IVF.

\section{Material and Methods}

A retrospective analysis was done between year 1999 and 2005, only patients with a history of primary infertility and with no other cause of infertility such as tubal factor or an ovulation were included. Couples were counselled for IVF, however if they opted for IUI their choice was respected.

Two groups were selected, the number of cases that had an IgG $>40 \%$ and IUI as a treatment option was 37 and underwent 56 cycles, this was consider as the first group. The second group was those who had IgG $>40 \%$ and had IVF as a treatment option were 19 and underwent 23 cycles ( as shown in table 1). Semen sample was collected by masturbation and examined accordingly to WHO criteria except for the morphology by the strict Tygerberg criteria. Detailed semen analysis was done for all samples on the day of the procedure including: volume of the ejaculate, color, viscosity, sperm density, motility, progressive motility, morphology. In addition to the immuno bead mixed Antiglobulin Reaction (MAR) test was used to detect IgG and IgA in the two groups.

\begin{tabular}{|l|l|l|}
\hline Category & Group I & Group II \\
\hline Number of Patients & 37 & 19 \\
\hline Number of Cycles & 56 & 23 \\
\hline
\end{tabular}

Table 1: Distribution of patients accordingly to the treatment option.

Gradient separation technique was used as the preparation method in both groups. For the first group, (0.2-1.0 ml) of washed sperm fraction was used for intrauterine insemination. For the other group, either IVF or ICSI procedure was done. I8 cycles were ICSI and only 5 had IVF. Only clinical pregnancies were considered into account [5]. 


\section{Results}

Data were expressed as a mean \pm SD. P- value of 0.05 or less was considered statistically significant. The mean BMI for Group I was $30.68 \pm 5.32$ and for Group II was $30.96 \pm 5.65$ with no statistical significant difference between the two groups (Table 2).

\begin{tabular}{|c|l|l|l|}
\hline Mean & $\begin{array}{l}\text { Group I (n=37) } \\
\text { Mean=SD Range }\end{array}$ & $\begin{array}{l}\text { Group II (n=19) } \\
\text { Mean=SD Range }\end{array}$ & P Value \\
\hline Mean BMI & $30.68 \pm 5.32$ & $30.96 \pm 5.65$ & 0.8352 \\
\hline
\end{tabular}

Table 2: BMI difference between the two Groups.

Out of Group I (those who had ovulation induction and IUI), 6 patients became pregnant giving a pregnancy rate (PR) per cycle of $11 \%$ and (PR) per patient of $16.2 \%$. On the other hand in Group II (those who had IVF), 10 patients conceived giving (PR) per cycle $43.5 \%$ and ( PR) per patient of 52.6\%. Which was statistically significant $(\mathrm{P}<0.05)$ (As Shown In Table 3).

\begin{tabular}{|l|l|l|l|}
\hline Pregnancy Rate & $\begin{array}{l}\text { Group I } \\
\text { IUI }\end{array}$ & $\begin{array}{l}\text { Group II } \\
\text { IVF/ICSI }\end{array}$ & P Value \\
\hline Preg./cycle & $6 / 56 \pm 11 \%$ & $10 / 23 \pm 43.5 \%$ & 0.0013 \\
\hline Preg./Pt. & $6 / 37 \pm 16.2 \%$ & $10 / 19 \pm 52.6 \%$ & 0.0046 \\
\hline
\end{tabular}

Table 3: Pregnancy rates between the two treatment options.

\section{Discussions}

It is generally accepted that the poor fertilization may be caused by the presence of sperm surface antibodies [5, 9], the immunobead test and the MAR test for IgG and IgA are the tests of choice for sperm surface antibodies [10] . In our study the MAR test for IgG and IgA was used to detect male immunological infertility. Although, IgA antibodies are more significant clinically, they rarely occur without association of IgG antibodies.

In-vitro studies in humans have demonstrated that spermatozoal autoantibodies induce a massive leukocytosis and sperm disruption at the level of the fundus uteri and the isthmic part of the oviduct [11]. In-vitro fertilization bypasses this adverse uterine reaction and has therefore become a very popular and effective treatment option for male infertility resulting from sperm surface antibodies [12]. Despite the fact that IVF results are very promising in male immunological cases, there is a strong evidence that ovarian hyperstimulation and IUI is a reasonable first treatment option in a substantial number of male infertility cases [13], theoretically IUI bypasses the barrier that cervical mucus poses to sperm coated with ASA as well washing the sperms. Our results indicate that, treatment with ovarian hyper stimulation and IUI is a valuable first choice method to use before starting the more invasive and expensive techniques of assisted reproduction. 


\section{Conclusion}

With the present limited knowledge of male immunological infertility, treatment with ovarian hyperstimulation and IUI affords pregnancy result of which patients should be counselled with before performing IVF/ICSI procedures. ICSI is the other form of ART to go for, especially to avoid the reduced or failed fertilization and the need of high concentration insemination with all its associated problems such as polyspermia and/or high (ROS) Reactive Oxygen Species.

\section{References}

1. Wallach EE. Reproductive Medicine and Surgery. Baltimore: Mosby year book (1995): 459-467.

2. Setu Mazumdor M.D, Adam S Levine M.D. Antisperm antibodies: etiology, pathogenesis, diagnosis, and treatment Fertility and Sterility. 70 (1998): 799-810.

3. Peters A.J, Coulam C.B. Review: sperm antibodies. Anti. Reprod. Immunol 27 (1992): 156-162.

4. Bronson R, Cooper G, Rosenfeld D. Sperm antibodies: their role in infertility. Fertil. Steril 42 (1984): 171183.

5. Shushan A, Schen Ker J.G. Immunological facts in Infertility. Am J. Reprod. Immunol 28 (1992): 285287.

6. Cunningham DS, Fulgham DL, Rayl DL, et al. [ antibodies to sperm surface antigens in women with genital tract infection. AmJ Obstet Gynecol 164 (1991): 791-796.

7. LiTS. Sperm immunology, infertility and fertility control. Obstet Gynecol 44 (1974): 607-623.

8. Adeghe J.H.A. Male sub fertility due to sperm antibodies: A clinical overview obstet. Gynecol 48 (1992): $1-8$.

9. Barratt C.L.R, Dunphy B.C, McLeod I, et al. The poor prognostic value of low to moderate levels of sperm surface-bound antibodies. Hum. Repro. 7 (1992): 95-98.

10. Andreou E, Mahmoud A, Vermeulen L. Comparison of different methods for the investigation of antisperm antibodies on spermatozoa, in seminal plasma and in serum. Hum. Repro 7 (1995): 95-98.

11. London S.N, Haney A.F, Weinberg J.B. Macrophages and infertility: Enhancement of human macrophagemediated sperm killing by antisperm antibodies. Fertil. Steril. 43 (1985): 274-278.

12. Rajah S.V, Paslow J.M, Howell R.J, et al. The effects on in-vitro fertilization of auto antibodies to spermatozoa in subfertile men. Hum. Repro 8 (1993): 1079-1082.

13. Ombelet W, Puttemans P, Bosmans E. Intrauterine insemination: A first step procedure in the algorithm of male subfertility treatment. Hum. Repro 10 (1995): 90-102.

Citation: Afaf Felemban MD, Seham M. Hassonah MD, Najla Felimban KD, Hadeel Alkhelb MD, Samar Hassan MD, Fahad Alsalman MD. Sperm Surface Antibodies: IUI vs. IVF Treatment. Obstetrics and Gynecology Research 1 (2018): 080-084.

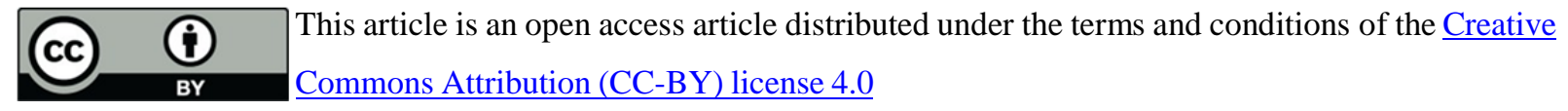

